This is an open access article distributed under the terms of the Creative Commons BY-NC-ND Licence

\title{
The homoeologous genes encoding C24-sterol methyltransferase 1 in Triticum aestivum: structural characteristics and effects of cold stress
}

\author{
A. RENKOVA ${ }^{1}$, J. VALITOVA ${ }^{1}$, H. SCHALLER ${ }^{2}$, and F. MINIBAYEVA ${ }^{1,3 *}$ \\ Kazan Institute of Biochemistry and Biophysics, FRC Kazan Scientific Center of RAS, Kazan 420111, Russia ${ }^{1}$ \\ Institute of Plant Molecular Biology, CNRS, F-67083 Strasbourg, France ${ }^{2}$ \\ Kazan (Volga Region) Federal University, Kazan 420008, Russia ${ }^{3}$
}

\begin{abstract}
A unique structural feature of plant sterols is the presence of a 24-alkyl group in the sterol side chain, which is synthesized by C24-sterol methyltransferase (SMT). Here we report for the first time that the bread wheat genome (AABBDD) contains at least three homoeologous genes encoding C24-sterol methyltransferase 1. While these copies have similar coding regions, they differ markedly in the nucleotide sequences of their non-coding regions. Sequencing de novo of the promoter regions of the TaSMT1 homoeologs demonstrated the occurrence of common and specific stress-sensitive ciselements such as LTR, the cis-element involved in low temperature response. These cis-elements, along with other factors, determine the differences in the effects of stress on the expression of homoeologous TaSMT1 genes. For example, TaSMT1-5A is constitutively expressed in the roots and leaves, while TaSMT1-4D gene is highly stress-responsive. Another important enzyme involved in sterol biosynthesis is C22-sterol desaturase, which converts $\beta$-sitosterol into stigmasterol. This enzyme is encoded by homoeologous TaCYP710A8 genes, which, in contrast to TaSMT1, are all up-regulated in response to stress. Cold-induced expression of TaCYP710A8 is greater in roots than in leaves. This may be due to the higher cold sensitivity of the roots and the necessity to increase the amount of stigmasterol known as a "stress sterol". Our findings suggest that the existence of homoeologous genes of sterol biosynthesis in polyploid plants supports the diversity of genetic mechanisms of sterol-mediated response of plants to stresses.
\end{abstract}

Additional key words: gene expression, C22-sterol desaturase, cis-elements.

\section{Introduction}

Sterols are essential structural elements of membranes and also components of signaling pathways, which are involved in the regulation of plant growth and development, and the responses of plants to stresses (Benveniste 2004). Plant membranes contain a complex mixture of sterols with $\beta$-sitosterol, stigmasterol, and campesterol as predominant species. Manipulation of sterols by binding and depletion greatly affects not only membrane permeability but also other biochemical and physiological characteristics in plants (Roche et al. 2008, Bonneau et al. 2010, Valitova et al. 2011, 2014). Sterols are isoprenoid derived lipids with a fused four-ring core structure and an aliphatic side chain (Benveniste 2004). A

unique structural feature of plant sterols is the presence of a 24-alkyl group in this side chain, the synthesis of which is catalyzed by S-adenosyl-L-Met-dependent C24-sterol methyltransferases (SMT). Alkylation happens in two steps where primary methylation is catalyzed by SMT1 (EC 2.1.1.142) resulting in the formation of methyl sterols (campesterol), and secondary methylation is catalyzed by SMT2 (EC 2.1.1.143) resulting in the formation of ethyl sterols ( $\beta$-sitosterol, stigmasterol).

The SMT is an integral protein localized in endoplasmic reticulum (Boutté and Grebe 2009). SMTs contain several conserved domains such as three sterol binding sites (Bouvier-Navé et al. 1997, Nes 2000) and

Submitted 5 December 2017, last revision 28 June 2018, accepted 29 June 2018.

Abbreviations: CDS - coding DNA sequences; LTR - low-temperature responsiveness; SMT - C24-sterol methyltransferase; qPCR - quantitative polymerase chain reaction; UTR - untranslated region.

Acknowledgments: This study was partly supported by the Russian Foundation for Basic Research (16-04-00676, 17-04-01562), the Research Fellowship from French Government (to AR) and partly performed according to the Russian Government Program of Competitive Growth of Kazan Federal University. We thank Richard Beckett (University of KwaZulu-Natal, South Africa) for useful discussions.

*Corresponding author; e-mail: minibayeva@kibb.knc.ru; fminibayeva@gmail.com 
S-adenosylmethionine binding site (Kagan and Clarke 1994) required for proper folding and functional activity (Nes et al. 2002). SMTs are present in plants, fungi, and protozoa but is absent in mammals (Nes 2000). Various plant species possess a diversity of SMT genes. For example, SMTs are encoded by three genes in Arabidopsis thaliana, three genes in Glycine max, four genes in Nicotina tabacum, and three genes in Oryza sativa (Bouvier-Navé et al. 1997, Schaller et al. 1998, Neelakandan et al. 2009, 2010, Carland et al. 2010). Some of these SMT genes have been cloned and their role in the regulation of 24-alkylsterol biosynthesis in plants has been characterized (Schaeffer et al. 2001, Neelakandan et al. 2009, Carland et al. 2010, Nakamoto et al. 2015).

Higher plants synthesize 24-methyl sterols and 24-ethyl sterols in defined proportions. Interestingly, in the Arabidopsis smt2 null mutant, the enzyme SMT1 can perform both primary and secondary methylation reactions to appropriate sterol substrates, although with different kinetics (Diener et al. 2000). Studies using mutants revealed that SMT plays roles in cell division, expansion, polarity and proliferation, embryogenesis, vein, shoot and root patterning, gravitropism, fertility, and hormone signaling (Diener et al. 2000, Schaeffer et al. 2001, Carland et al. 2002, Willemsen et al. 2003, Souter et al. 2004, Men et al. 2008, Nakamoto et al. 2015). Several studies have demonstrated that plant sterols, particularly $\beta$-sitosterol, possess antioxidant activity (Vivancos and Moreno 2005, Li et al. 2007, Paniagua-Pérez et al. 2008, Posé et al. 2009). Arabidopsis mutants with an upregulated expression of SMT genes and an increased content of $\beta$-sitosterol showed higher resistance to oxidative stress compared to the wild type (Wang et al. 2012). Thus, SMT is a key enzyme in plant sterol biosynthesis, whose functioning provides the optimal balance of molecular species of sterols during plant ontogenesis and stress response.

In Triticum aestivum, a single gene encoding SMT1 has been annotated so far (GenBank: U60755.1; Subramaniam et al. 1999). Information about sequences and structure of other SMT genes in T. aestivum is still unknown. Wheat is probably the most important crop in the world, yet it has one of the most challenging genomes. Bread wheat is an allohexaploid consisting of seven groups of chromosomes, each group containing a set of three homoeologous chromosomes belonging to the A, B, and D genomes, derived from a common ancestor (Marcussen et al. 2014). Thus, three representatives of each single copy of a gene are present in the wheat genome. They are referred to as homoeologous genes. The large size and intricate organization of the wheat genome hamper both the genome-wide sequencing and gene annotation.

Another enzyme involved in sterol biosynthesis is C22sterol desaturase, or CYP710A8 from the plant cytochrome $\mathrm{P}_{450}$ subfamily, which converts $\beta$-sitosterol into stigmasterol (Morikawa et al. 2006, Arnqvist et al. 2008). Stigmasterol in most higher plants and ergosterol in most fungi and some algae have a double bond at C22. This double bond is formed by a desaturation reaction, which is the final stage of plant sterol biosynthesis (Ohta and Mizutani 2013). Earlier, full-length cDNAs of wheat CYP710A8 genes were isolated and mapped on chromosome 3A, 3B, and 3D (Tang et al. 2011).

We hypothesize the existence of homoeologous SMT1 genes in polyploid wheat and suggest that they are involved in a variety of sterol-mediated responses to stresses. Therefore, the aim of the present study was to sequence and analyze the structure and expression of homoeologous TaSMT1 genes. For this purpose, we firstly determined the DNA sequence of the three homoeologous TaSMT1 genes in wheat. The structures of the gene regulatory and coding regions were analyzed. Secondly, we studied the effect of cold stress on the expression of TaSMT1 and TaCYP710A8 genes in the roots and leaves of wheat seedlings. Earlier it was shown that cold stress can induce the overexpression of a number of enzymes of sterol biosynthesis in cereals, which increases the amount of membrane sterols (Uemura and Steponkus 1994). Thirdly, the upstream regions of the homoeologous TaSMT1 genes were sequenced and potential stress regulatory motifs were analyzed in silico.

\section{Materials and methods}

Plants and cultivation: Wheat (Triticum aestivum L. cv. Kazanskaya Jubileinaya; Niva Tatarstana, Kazan, Russia) seedlings were grown hydroponically in $0.25 \mathrm{mM} \mathrm{CaCl}_{2}$ solution in a growth chamber at a temperature of $25^{\circ} \mathrm{C}$., a 12-h photoperiod, and an irradiance of $460 \mu \mathrm{mol} \mathrm{m}^{-2} \mathrm{~s}^{-1}$ for $5 \mathrm{~d}$. To induce cold stress, 5-d-old seedlings were transferred to $4{ }^{\circ} \mathrm{C}$ for 1 and $12 \mathrm{~h}$.

Isolation of plant DNA and molecular cloning of target gene fragments: Genomic DNA from $T$. aestivum seedlings was isolated using cetyltrimethyl ammonium bromide (CTAB) buffer. DNA concentration and quality

was evaluated spectrophotometrically using NanoDrop ND-1000 spectrophotometer (Thermo Scientific, Waltham, USA) and $1 \%(\mathrm{~m} / \mathrm{v})$ agarose gel electrophoresis. For the amplification of the fragments of homoeologous TaSMT1 genes, primers were constructed on the conserved regions of TaSMT1. To amplify promoter regions of homoeologous TaSMT1, gene-specific primers were designed based on their sequencing data from IWGSC database. Forward and reverse primers are listed in Table 1 Suppl. PCR was performed by means of C1000 Touch ${ }^{T M}$ thermal cycler (Bio-Rad, Hercules, USA) using genomic DNA, primers and polymerases Maxima Hot Start Taq 
DNA (Thermo Scientific) and GoTaq Flexi DNA (Promega, Madison, USA).

For promoter sequencing, the PCR was conducted with a Genome Walker universal kit (Clontech, Mountain View, USA) using the genomic DNA as template following the manufacturer instructions. Genomic DNA from T. aestivum was digested with four blunt end cutting enzymes (DraI, EcoRV, PvuII, StuI) to construct the DNA libraries. DNA fragments were further purified and ligated to the Genome Walker adaptor provided in the kit. A primary PCR was performed with the outer adaptor primer (AP1) and the corresponding outer TaSMT1-gene specific primer to walk in the $5^{\prime}$ end direction (GSP1). The product of this primary PCR was then diluted (1:50) and used as a template for a second walk using the nested adaptor primer (AP2) and one of the TaSMT1-gene-specific primers (GSP2). Using the GSP2 primer, the purified PCR products were then sequenced. The gene-specific oligo sequences used in PCR amplification are as follows: GSP1: 5'-CGGTGTAGTTGGATTTCCTCGATTCC TCC-3'; GSP2: 5'-GCAACAGTACAGGGAATGAAG ACACTCGGC-3'.

PCR products were purified using Axy Prep $^{\mathrm{TM}}$ PCR cleanup kit (Axygen Biosciences, Union City, USA) and cloned into the $p G E M^{\mathbb{B}}-T$ Easy vector system (Promega) according to the manufacturer protocol. Nucleotide sequences were determined using an automatic $A B I 3130$ genetic analyzer (Applied Biosystems, Foster City, USA) using Big Dye Terminator v.3.1 cycle sequencing kit (Applied Biosystems).

Analysis of gene expression by real-time quantitative PCR: Total RNA was isolated from wheat using RNeasy Plant Mini kit (Qiagen, Hilden, Germany) according to the manufacturer protocols. RNA concentration and purity were assessed using NanoDrop ${ }^{\circledR} N D-1000$ spectrophotometer and the integrity was verified by $1 \%$ agarose gel electrophoresis. The reverse transcription (RT) reaction was performed using C1000 Touch $^{\mathrm{TM}}$ thermal cycler with reverse transcriptase RevertAid RT (Thermo Scientific) and Oligo(dT) $)_{16}$ primers (Sintol, Moscow, Russia) according to the manufacturer protocol.

Real-time qPCR was performed using an ICycler IQ 2 Multicolor real-time PCR detection system (Bio-Rad). The templates were amplified three times at $95{ }^{\circ} \mathrm{C}$ for $3 \mathrm{~min}$ followed by 40 cycles of amplification $\left(94{ }^{\circ} \mathrm{C}\right.$ for $10 \mathrm{~s}$ and $55 / 60{ }^{\circ} \mathrm{C}$ for $\left.40 \mathrm{~s}\right)$. ADP-ribosylation factor (TaARF) and RNase L inhibitor-like protein ( $T a R L I)$ genes were used as reference genes (Paolacci et al. 2009). The gene-specific primers and TaqMan probes used for real-time qPCR are listed in Table 1 Suppl. For TaCYP710A8 genes, previously published primers were used (Tang et al. 2011). Quantification was performed according to Pfaffl (2001).

Bioinformatic analysis: Nucleotide sequences and chromosomal localization of the TaSMT1 genes were determined using the BLAST facility of the international wheat genome sequencing consortium database (IWGSC) (http://www.wheatgenome.org/) hosted by URGI server (http://urgi.versailles.inra.fr). The complete sequences of A, B and D TaSMTl genes of hexaploid wheat were obtained following the initial query during the survey of the coding DNA sequences (CDS) of $T$. aestivum $\triangle$-24-sterol methyltransferase mRNA (NCBI accession U60754; GenBank: U60754.1). Search and comparison of homologous sequences was performed using the BLAST program at the NCBI server. Design of gene-specific primers, translation of CDS, sequence alignment, and estimation of molecular mass of predicted protein was performed using the program Vector NTI Suite 9 (Invitrogen, Carlsbad, USA). Exon-intron structures of TaSMT1-5A, TaSMT1-4B, and TaSMT1-4D genes were predicted by using online mRNA-to-genomic alignment program Spidey (http://www.ncbi.nlm.nih.gov/spidey/). The phylogenetic tree of selected $S M T$ s was constructed by the bootstrap test (1 000 replicates) using MEGA7 software.

The promoter sequences were analyzed in silico for the presence of potential cis-acting elements using PLANTCARE (http://bioinformatics.psb.ugent.be/ webtools/plantcare/html/) (Lescot et al. 2002) and PLACE databases (Higo et al. 1999). The database of $\mathrm{CpG}$ islands and analytical tool (DBCAT) (http://dbcat.cgm.ntu. edu.tw/) were applied to recognize comprehensive methylation profiles of DNA alteration in the promoter sequences.

Statistics: All experiments were performed with at least three biological and four analytical replicates. Data presented are means and standard errors. Statistical analysis was performed using the two-tailed Student's $t$-test in Microsoft Excel 2013.

\section{Results}

The full-length coding regions of three homoeologous genes of wheat $S M T 1$ were found using URGI database. These genes are located on the long arms of $5 \mathrm{AL}, 4 \mathrm{BL}$, and 4DL chromosomes and were named as TaSMT1-5A, TaSMT1-4B and TaSMT1-4D, respectively. The nucleotide sequences of TaSMT1 homoeologous genes were confirmed experimentally by several rounds of amplifications and cloning with specific pair of primers.

Multiple alignments of TaSMT1 clones suggested the presence of several products in the PCR amplification mix. The sequenced fragments corresponded to the putative sequences from the URGI database from different wheat genomes (A, B, and D). A comparison analysis of the genomic sequences of the three TaSMT1 genes with their corresponding cDNA sequences showed that these genes are characterized by structural similarities and consist of 
twelve (TaSMT1-5A), ten (TaSMT1-4B), and eleven (TaSMT1-4D) exons, with all the splicing sites complying with the GT-AG rule (Fig. 1A). Analysis allowed us to identify four conserved exons with identical sequences and sizes among three homoeologous SMT1 genes from wheat and SMT1 genes from other plants (Fig. 1A). Comparative analysis of the CDS of the three TaSMT1 genes confirmed that they had a high degree of similarity: $96.5 \%$
(TaSMT1-5A vs TaSMT1-4B), $97.7 \%$ (TaSMT1-5A vs TaSMT1-4D), and 97.6\% (TaSMT1-4B vs TaSMT1-4D) (Table 1). Despite these similarities in CDS, there are small differences due to insertions/deletions and single nucleotide polymorphisms (Fig. 1B). Differences in the sizes of the intron sequences among three homoeologous SMT1 genes in wheat were also observed (Fig. 1A).

A
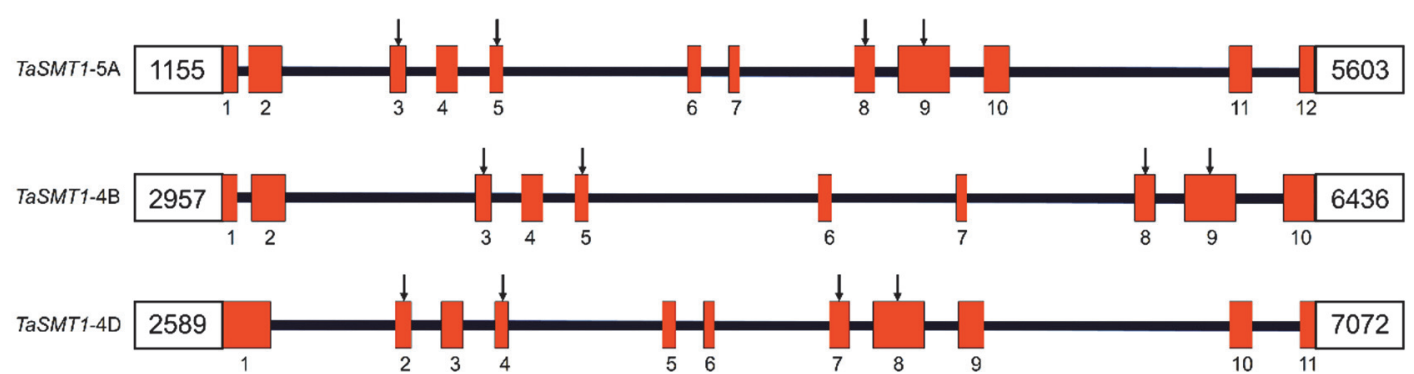

B

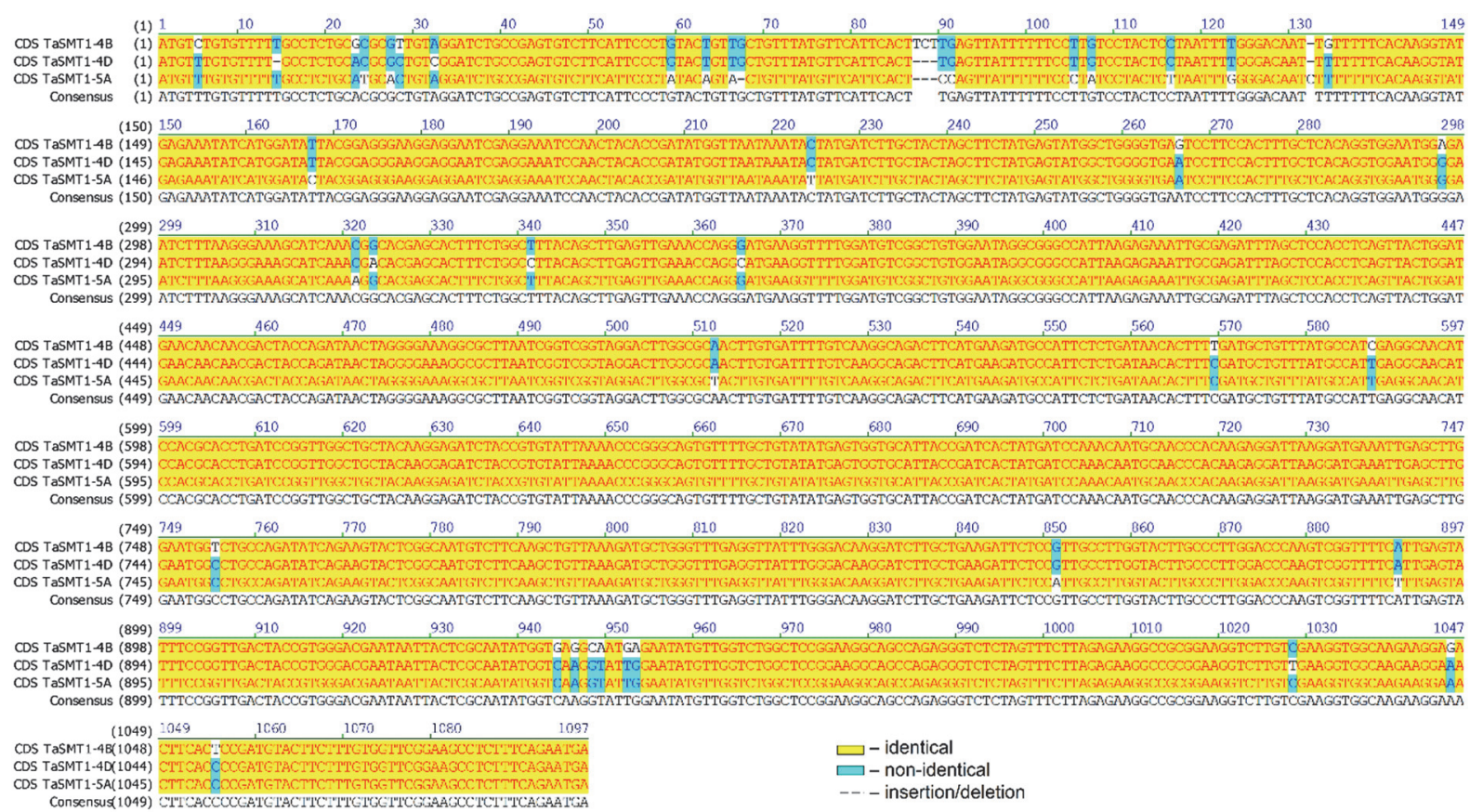

Fig. 1. A - Schematic representation of the structure of three homeologous TaSMT1 genes of wheat. The rectangles show exons, the lines show introns, and the arrows show the conserved exons (http://www.ncbi.nlm.nih.gov/spidey/). At the 5'- and 3' ends of the splicing site, the introns have conserved base pairs (GT ... AG). $B$ - Alignments of the coding DNA sequence (CDS) of three homoeologous TaSMT1 genes using Vector NTI program.

Table 1. Identity of TaSMT1 sequences.

\begin{tabular}{llll}
\hline Alignment & Identity [\%] & & \\
& TaSMT1-5A/4B & TaSMT1-5A/4D & TaSMT1-4B/4D \\
\hline Genomic DNA sequence & 54.7 & 74.3 & 70.5 \\
CDS & 96.5 & 97.7 & 97.6 \\
Promoter sequence & 32.6 & 37.3 & 81.1 \\
\hline
\end{tabular}




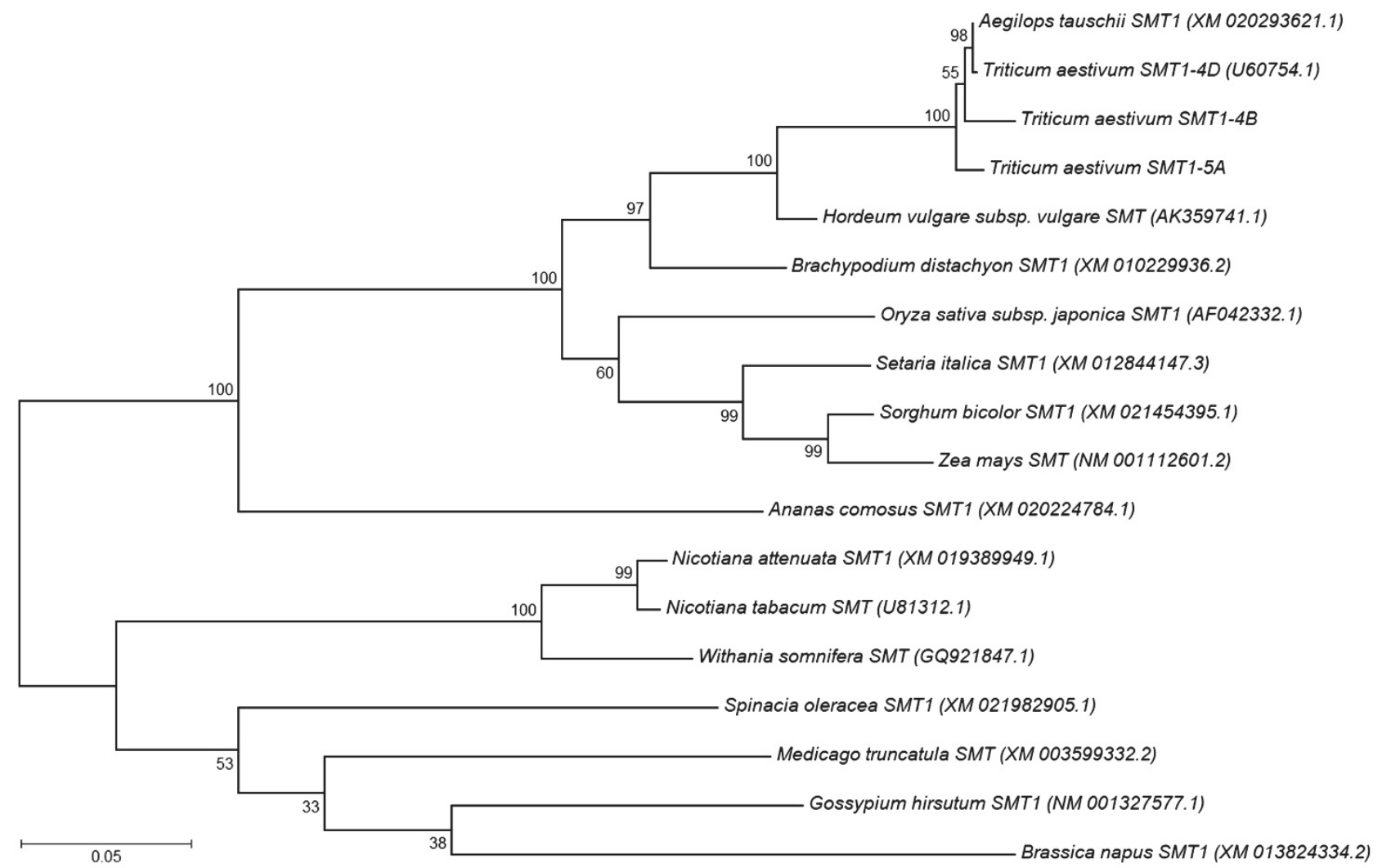

Fig. 2. Phylogenetic tree of the CDS of SMT gene family from various plant species constructed by the bootstrap test using $M E G A 7$ software. The numbers indicate confidence values.

A phylogenetic tree was constructed on the basis of the analysis of CDS of SMT1 genes in wheat and other plant species. The TaSMT1 sequences of wheat, as expected, were most similar to SMT1 of cereals such as Aegilops and barley (Fig. 2). Furthermore, comparison of the full-length deduced amino acid sequences of mature TaSMT1 protein from wheat with those of protein sequences of other SMT1 demonstrated that these sequences were highly conserved among the other plant species and yeasts (Table 2 Suppl.). TaSMT1 shared the highest similarity with SMT1 from barley, Brachypodium, and Aegilops with similarities of 98,96 , and $94 \%$, respectively, and the least similarity with SMT1 from Saccharomyces cerevisiae.

SMTs contained several conserved domains such as three sterol binding sites and S-adenosyl methionine binding site required for proper folding and functional enzyme activity (Fig. 3). Important amino acid residues Glu $_{82}$, His 90, Asp $_{125}, \mathrm{Glu} / \mathrm{Asp}_{152}, \mathrm{Glu}_{195}, \mathrm{Glu}_{224}, \mathrm{Asp}_{276}$, which are directly involved in the binding sterols and S-adenosyl methionine to the enzyme via hydrogen bonds (Nes et al. 2004) were found in TaSMT1.

In order to characterize the expression of different copies of the TaSMTl gene, we estimated the relative transcriptions of TaSMT1-5A, TaSMT1-4B, and TaSMT1-4D genes in the roots and leaves of control wheat seedlings and seedlings stressed with a low temperature $\left(4{ }^{\circ} \mathrm{C}\right)$. PCR analysis followed by electrophoretic DNA separation in agarose gel revealed the presence of TaSMT1-5A and TaSMT1-4D transcripts in control plants both in the roots and leaves. Unfortunately, we were unable to quantify the TaSMT1-4B transcript in the roots or the leaves, probably because TaSMT1-4B was silent or inactive. Expression of TaSMT1-5A was higher in the leaves (Fig. 4A), whereas the TaSMT1-4D gene was more strongly expressed in the roots (Fig. $4 B$ ). In addition, the expression of the TaSMT1-5A gene in roots and leaves at $4 \quad{ }^{\circ} \mathrm{C}$ remained practically unchanged (Fig. 4A). Conversely, cold increased the expression of the TaSMTI4D gene in both the roots and leaves, with the greatest (4-fold) changes observed in roots exposed to cold for $12 \mathrm{~h}$.

The effect of cold stress on the expression of homoeologous genes encoding C22-sterol desaturase, or CYP710A8, was analyzed in the roots and leaves of wheat seedlings. Using specific primers for TaCYP710A8-3A and common primers for TaCYP710A8-3B and TaCYP710A8-3D homoeologous genes, which share $97.8 \%$ identity, we estimated the expression of TaCYP710A8 in control plants and also after short-term $(1 \mathrm{~h})$ and long-term $(12 \mathrm{~h})$ exposure to $4{ }^{\circ} \mathrm{C}$ (Fig. 5). Analysis of gene expression demonstrated co-expression of CYP710A8 homoeologous genes in the roots and leaves. In the roots of cold-treated wheat seedlings, the expression of all genes steadily increased (Fig. 5). Conversely, in the leaves of cold-treated seedlings, the expression profile of 
TaCYP710A8 genes was characterized by initial upregulation followed by down-regulation after longer exposure to cold (Fig. 5).
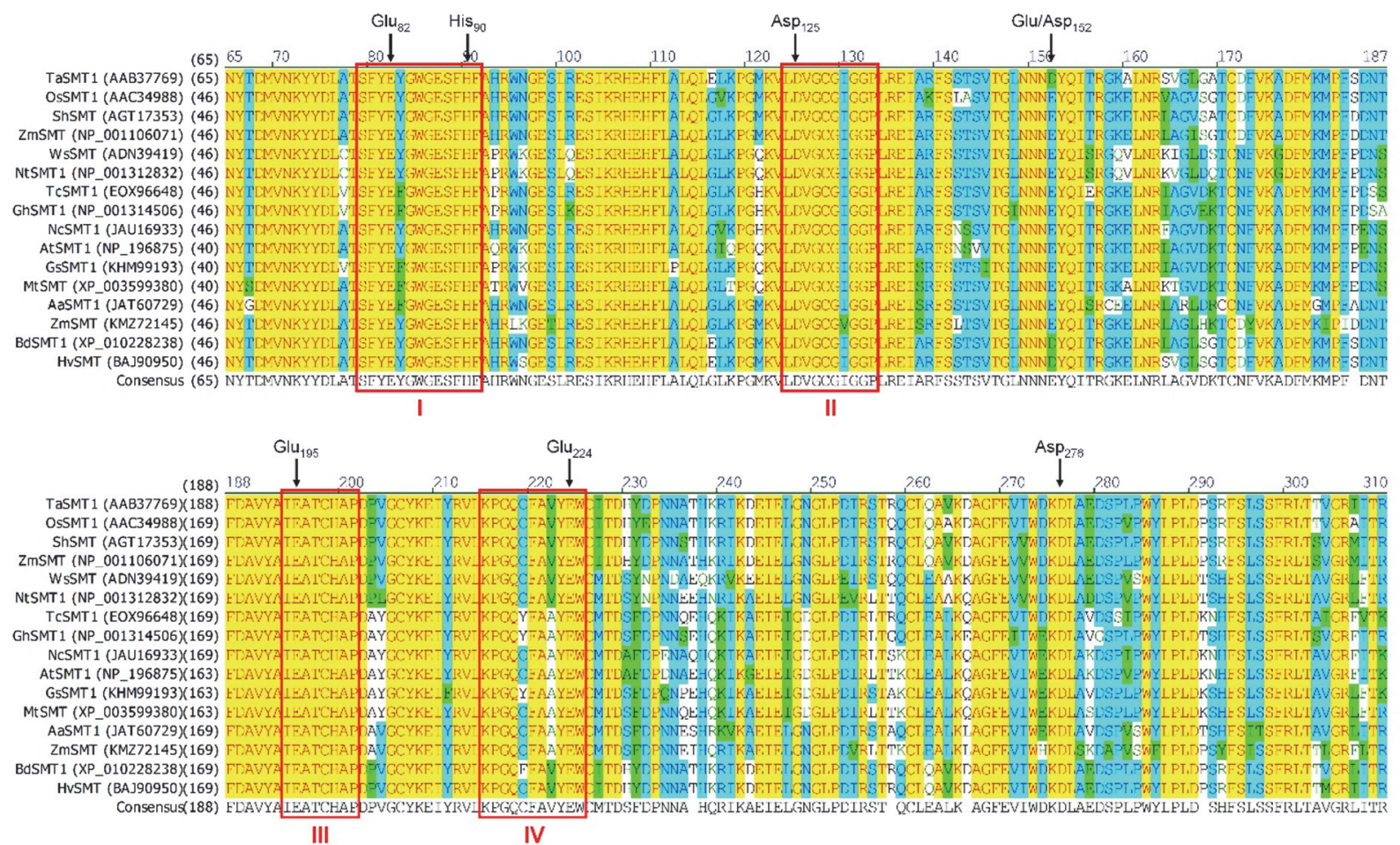

Fig. 3. Alignments of the deduced amino acid sequences of the SMT1 using Vector NTI program. The rectangles show conserved domains of SMT1: I, III, and IV are sterol binding sites; II - AdoMet binding site. The conserved amino acid residues, which are directly involved in the binding sterols and adenosyl methionine to the enzyme via hydrogen bonds: Glu82, His90, Asp 125 , Glu/Asp 152 , $\mathrm{Glu}_{195}, \mathrm{Glu}_{224}$, and Asp 276 are indicated with arrows.

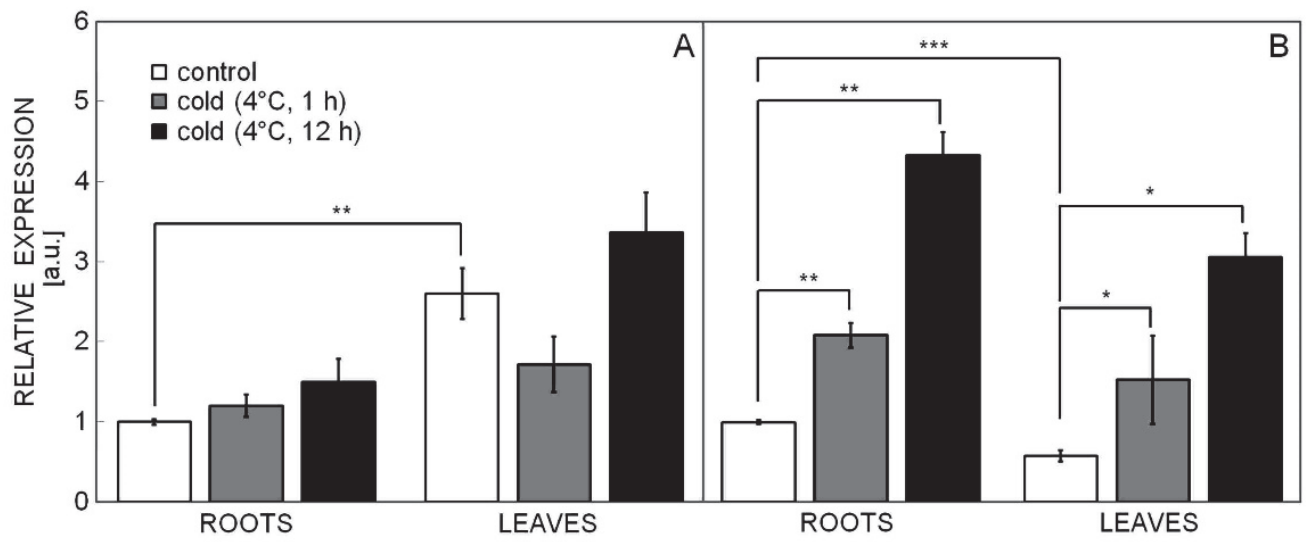

Fig. 4. Relative expressions of TaSMT1-5A $(A)$ and TaSMT1-4D $(B)$ genes assessed with real-time qPCR in wheat roots and leaves treated with cold stress. One, two, or three asterisks represent significant difference at $P \leq 0.05, P \leq 0.01$, and $P \leq 0.001$, respectively (two-tailed Student's $t$-test; $n=3-6$ ).

Differential expression of wheat homoeologous genes TaSMT1-5A and TaSMT1-4D in response to cold treatment may depend on the presence of specific sequences in the promoter structure. Therefore, the $1-1.5$ $\mathrm{kb}$ upstream regions to the ATG initiation codon of TaSMT1-5A, TaSMT1-4B, and TaSMT1-4D were sequenced. The alignment of promoter sequences of TaSMT1 homoeologues revealed differences in the upstream regions of these genes. An alignment using Vector software showed that the homology in upstream regions were as following: $32.6 \%$ (TaSMT1-5A vs TaSMT1-4B), $37.3 \%$ (TaSMT1-5A vs TaSMT1-4D) and $81.1 \%$ (TaSMT1-4B vs TaSMT1-4D) (Table 1). Potential cis-acting elements were identified based on their homology to previously characterized regulatory motifs in plants, as documented in PLACE and PLANTCARE 
databases (Higo et al. 1999, Lescot et al. 2002) and listed in Table 3 Suppl. Analysis of the sequenced promoter regions of TASMT1 genes showed that they contain conserved sites (TATA-box and CAAT-box) and the following stress-sensitive motifs: cis-elements induced by light (ACE, AE, and G-box), drought (MBS), low and high temperatures (LTR and HSE), and hormones such as ABA (ABRE), gibberellin (GARE and P-box), ethylene (ERE), salicylic acid (TCA element), auxin (TGA element), and methyl jasmonate (TGACG motif) (Table 3 Suppl.).

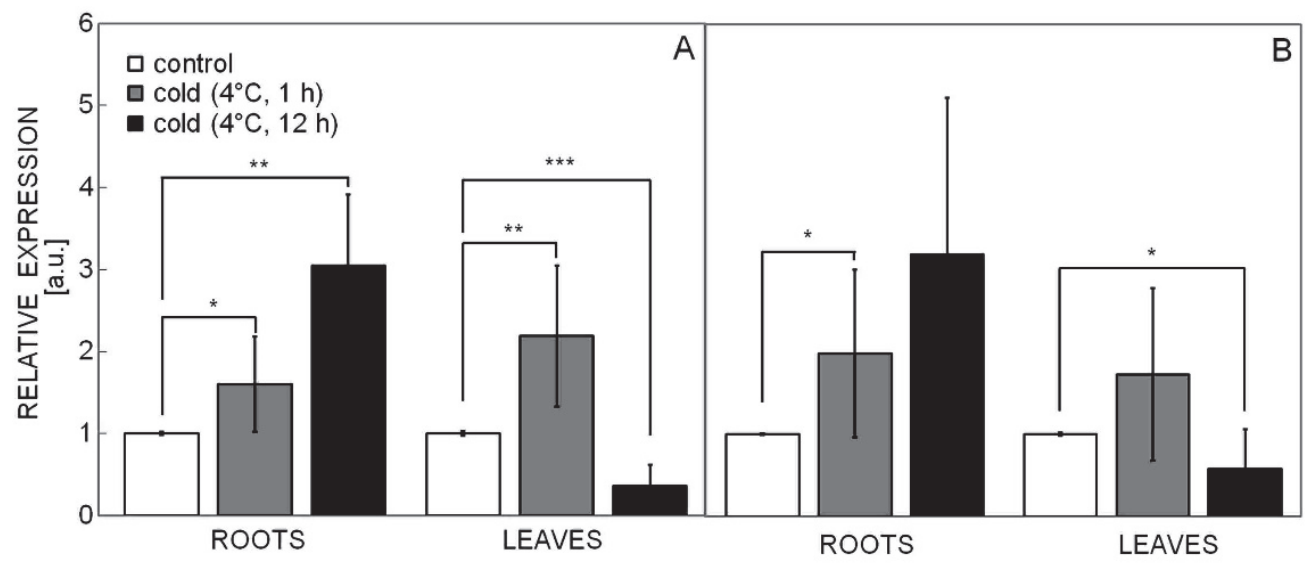

Fig. 5. Relative expression of $T a C Y P 710 A 8-3 \mathrm{~A}(A)$ and $T a C Y P 710 A 8-3 \mathrm{~B} /-3 \mathrm{D}(B)$ genes assessed with real-time qPCR in wheat roots and leaves treated with cold stress. One, two, or three asterisks represent significant difference at $P \leq 0.05, P \leq 0.01$, and $P \leq 0.001$, respectively (two-tailed Student's $t$-test; $n=3-6$ ).

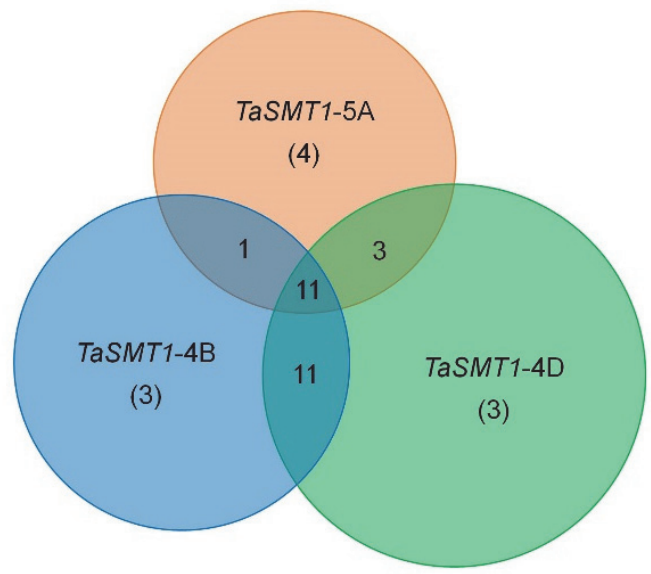

Fig. 6. Venn diagram illustrating the distribution of common (in intersections) and specific (in brackets) potential cis-elements in the promoter regions of three homoeologous TaSMT1 genes.

\section{Discussion}

The ability to synthesize C24 alkyl sterols and more specifically C24 ethyl sterols is unique to the plant kingdom (Neelakandan et al. 2009, Nakamoto et al. 2015). The SMT1 gene catalyzes the first step towards the synthesis of C24 alkyl sterols (Carland et al. 2002, Willemsen et al. 2003). In work reported here we showed that the bread wheat genome contains at least three homoeologous genes encoding C24-sterol methyltransferase 1. We also demonstrated different patterns of expression of TaSMT1 and TaCYP710A8 genes in roots and leaves in control conditions as well during cold stress.
Fig. 6 illustrates the distribution of common and specific potential cis-elements in the promoter regions of TaSMT1-5A vs TaSMT1-4B, TaSMT1-5A vs TaSMT1-4D, and TaSMT1-4B vs TaSMT1-4D as a Venn diagram. Eleven common cis-elements were predicted within the TaSMT1 promoter regions. These included conserved sites (TATA-box, CAAT-box, and CCAATbox), stress-sensitive motifs induced by anaerobiosis, light, drought, and developmentally regulated motifs such as GARE involved in gibberellin responsiveness and Skn-1 for expression in the endosperm (Table 3 Suppl.). In addition, the presence of $\mathrm{CpG}$ islands in the promoter regions of TaSMT1 genes was evaluated using the database of $\mathrm{CpG}$ islands and analytical tool (DBCAT). Analysis of the 1 to $3-\mathrm{kb}$ upstream regions of TaSMT1 genes showed that $\mathrm{CpG}$ islands are present in the promoter sequences of TaSMT1-4BL and TaSMT1-4DL (Fig. 1 Suppl.).

Furthermore, we suggested that LTR, the cis-element involved in low temperature responsiveness, which we found in the TaSMT1-4D gene, together with other stressresponsive motifs in the promoter was involved in the regulation of TaSMT1 expression in response to cold treatment. These findings suggest that the existence of homoeologous genes of sterol biosynthesis in polyploid plants supports the diversity of genetic mechanisms of sterol-mediated response of plants to stresses.

It was suggested that SMT lineages are likely concurrent with the origin of the eukaryote domain, and 
then lost variably in some lineages because no SMT homologs from advanced animals (above the sponges) were identified (Haubrich et al. 2015). The phylogenetic tree of the SMT1 family demonstrated that three wheat SMT1s showed the highest similarity (more than $98 \%$ ) with Aegilops tauschii, other cereals species also showed high identity with wheat (Fig. 2). Therefore, the sequence similarity exists between wheat SMT and typical SMT gene family from other plant species. Furthermore, analysis of the full-length deduced amino acid sequences of mature TaSMT1 protein demonstrated that highly conserved regions correspond to substrate binding segments for sterol and S-adenosyl methionine binding site (Fig. 3) designated as regions I, III, and IV and region II according to Nes et al. 2004.

Three homoeologous TaSMT1 genes were located on the $5 \mathrm{~A}, 4 \mathrm{~B}$, and $4 \mathrm{D}$ chromosomes of the hexaploid wheat genome. Interestingly, two genes (TaSMT1-4B and TaSMT1-4D) were located on homoeologous group 4 chromosomes and one gene (TaSMT1-5A) was located on homoeologous group 5 chromosomes. Homoeologous genes are usually located on the same group of chromosomes. The pattern of chromosome arm locations showed that genes located on 5AL chromosome could originally have been derived from the 4AL chromosome. Possibly during the evolution of modern-day chromosome $4 \mathrm{~A}$ a translocation event occurred involving the distal regions of the long arm of chromosomes $4 \mathrm{~A}$ and $5 \mathrm{~A}$. The non-homoeologous 4AL/5AL translocation occurred at the diploid level, e.g., in T. monococcum (Devos et al. 1995, Ma et al. 2013). Various chromosome translocations have also been reported for other wheat genes, such as TaEPSPS (TaEPSPS-7A1, TaEPSPS-4A1, and TaEPSPS-7D1) (Aramrak et al. 2015) and Waxy genes (Wx-7A, $W x-4 \mathrm{~A}$ and $W x$-7D) (Huang et al. 2010).

Comparison of SMT genomic structures from different taxa indicates that the SMT genes consist of three major classes based on their intron pattern. Class 1 or algal SMT1s have five introns, class 2 or vascular plant SMT1s have five to twelve introns and class 3, comprising both SMT2s of plants and SMT1s of non-photosynthetic organisms, have no introns (Neelakandan et al. 2009). The $S M T$ genes in wheat all have structural similarities and consist of twelve (TaSMT1-5A), ten (TaSMT1-4B), and eleven (TaSMT1-4D) exons, and are similar to homologous SMT1 genes in other plants. An analysis of gene structure revealed that these genes include four conserved exons with identical sequences and sizes (Fig. 1A). It is likely that that these exons encode functionally important domains of the SMT1 protein in plants. Translation of the nucleotide sequence of these exons into the amino acid sequence confirmed that these exons encode sterol-binding sites, which are required for the functional activity of the protein. Thus, based on their introns and exons, TaSMT1 genes correspond to the structure of the homologous genes of the SMT1 family of higher plants (Neelakandan et al. 2009).
The alignment of the CDS revealed the presence of polymorphic sites where nucleotide substitutions and deletions occurred (Fig. 1B). These differences in nucleotide sequences might allow specificity of gene functioning. There may be different evolutionary consequences of the structural and functional divergence of homoeologous genes in polyploids. Possible outcomes include first subfunctionalization, i.e., separation of functions between copies (co-expression of genes, inhibition/stimulation of transcription of certain homoeologs, as well as tissue-, organ-, and stage-specific expression). Second, neofunctionalization can occur, where one gene may acquire new functions. Finally, in pseudogenization there is complete loss of function by one of the homoeologs, and it subsequently becomes a pseudogene (Wendel 2000). Polyploid organisms have the advantage that they can display structural and functional divergence of homoeologous genes, which can increase the adaptive potential of the organism in a changing environment.

In wheat, the three related TaSMT1 homoeologs possess conserved exon/intron structure and CDS but contain significant sequence variations within non-coding regions. These variations may result in the differences in gene expression. Earlier, we demonstrated that cold stress greatly changes the sterol content of wheat roots (Sulkarnayeva et al. 2014). In the present work, we found that cold stress upregulated the TaSMT1-4D gene expression in both the roots and the leaves (Fig. 4B), which might facilitate the acclimation of membranes to cold. The role of TaSMT1 genes in the response of plants to stress was further supported by the presence of potentially stress responsive cis-elements in the promoter region of homoeologous TaSMT1 genes. The promoter regions contained several cis-regulatory elements related to the response to biotic and abiotic stresses and hormonal stimuli (Table 3 Suppl.). It is known that the regulatory elements ABRE, LTR, MBS, G-box, and CBF/DREB transcription factors are also present in the promoters of other genes whose expression is induced by cold stress (Fowler and Thomashow 2002, Ruelland et al. 2009). Interestingly, the cold-sensitive cis-element LTR was present in the promoter regions of TaSMT1-4B and TaSMT1-4D but not in TaSMT1-5A. We suggested that LTR cis-element can influence the expression of TaSMT14D in response to cold stress, but the expression of TaSMT1-4B was not cold stimulated. This indicated complex regulation of the activity of these homoeologous genes, and thus more work is needed to unravel the fine mechanisms governing the activity of TaSMT1 in a polyploid organism. Furthermore, the presence of $\mathrm{CpG}$ island, which was found in the promoter sequences of TaSMT1-4D (Fig. 1 Suppl.) might contribute to the stable expression of this gene, as $\mathrm{CpG}$ islands usually remain unmethylated (Ashikawa 2001, Papadakis et al. 2004). Despite the fact that $\mathrm{CpG}$ islands were not found in the promoter of TaSMT1-5A, it cannot be ruled out that an 
increase in the length of sequenced region will give us more information about the presence of $\mathrm{CpG}$ islands.

Analysis of the expression of homoeologous genes encoding C22-sterol desaturase (CYP710A8), another enzyme involved in sterol biosynthesis, showed different patterns of co-expression of these genes in the roots and leaves. In the roots of cold-treated wheat seedlings, the expression of all genes steadily increased over the timecourse of cold exposure, while leaves displayed a bellshaped response, with gene up-regulation during early stress response and down-regulation after longer exposure to cold (Fig. 5). Thus, our data demonstrated that the expression of homoeologous TaCYP710A8 genes differed in the roots and leaves. Considering that $\mathrm{C} 22$-sterol desaturase converts $\beta$-sitosterol into stigmasterol, continuous up-regulation of $\mathrm{TaCYP} 710 \mathrm{~A} 8$ genes in roots supports the higher cold sensitivity of roots than leaves as demonstrated by Zhang et al. (2017) and the necessity to raise the content of stigmasterol known as a "stress sterol".

\section{References}

Ashikawa, I.: Gene-associated $\mathrm{CpG}$ islands in plants as revealed by analyses of genomic sequences. - Plant J. 26: 617-625, 2001.

Aramrak, A., Kidwell, K.K., Steber, C.M., Burke, I.C.: Molecular and phylogenetic characterization of the homoeologous EPSP synthase genes of allohexaploid wheat, Triticum aestivum (L.). - BMC Genomics 16: 844, 2015.

Arnqvist, L., Persson, M., Jonsson, L., Dutta, P.C., Sitbon, F.: Overexpression of CYP710A1 and CYP710A4 in transgenic Arabidopsis plants increases the level of stigmasterol at the expense of sitosterol. - Planta 227: 309-317, 2008.

Benveniste, P.: Biosynthesis and accumulation of sterols. - Annu. Rev. Plant Biol. 55: 429-457, 2004.

Bonneau, L., Gerbeau-Pissot, P., Thomas, D., Der, C., Lherminier, J., Bourque, S., Roche, Y., Simon-Plas, F.: Plasma membrane sterol complexation, generated by filipin, triggers signaling responses in tobacco cells. - Biochim. biophys. Acta 1798: 2150-2159, 2010.

Boutté, Y., Grebe, M.: Cellular processes relying on sterol function in plants. - Curr. Opin. Plant Biol. 12: 705-713, 2009.

Bouvier-Navé, P., Husselstein, T., Desprez, T., Benveniste, P.: Identification of cDNAs encoding sterol methyl-transferases involved in the second methylation step of plant sterol biosynthesis. - Eur. J. Biochem. 246: 518-529, 1997.

Carland, F., Fujioka, S., Nelson, T.: The sterol methyltransferases SMT1, SMT2, and SMT3 influence Arabidopsis development through nonbrassinosteroid products. - Plant Physiol. 153: 741-756, 2010.

Carland, F., Fujioka, S., Takatsuto, S., Yoshida, S., Nelson, T.: The identification of CVP1 reveals a role for sterols in vascular patterning. - Plant Cell 14: 2045-2058, 2002.

Devos, K.M., Dubcovsky, J., Dvořák, J., Chinoy, C.N., Gale, M.D.: Structural evolution of wheat chromosomes 4A, 5A, and $7 \mathrm{~B}$ and its impact on recombination. - Theor. sppl. Genet. 91: 282-288, 1995.

Diener, A.C., Li, H., Zou, W.X., Whoriskey, W.J., Nes, W.D.,
In conclusion, this is the first report that shows that allohexaploid wheat possesses three homoeologous TaSMT1 genes located on chromosomes A, B, and D. Despite significant similarities in their coding regions, the nucleotide sequences of the non-coding regions markedly differed. The presence of specific stress-sensitive ciselements in their promoter regions, along with other factors, determined the differences in the patterns of expression of the TaSMT1 genes: only the TaSMT1-4D gene was highly responsive to cold stress. Conversely, expression of the homoeologous TaCYP710A8 genes changed in the same way in response to cold stress. These findings suggest that the existence of homoeologous genes of sterol biosynthesis in polyploid plants supports the diversity of epigenetic mechanisms of sterol-mediated response of plants to stresses. New data obtained will allow us in future to develop novel experimental tools of increasing stress tolerance and sustainability of crops by targeted regulation of sterol metabolism.

Fink, G.R.: STEROL METHYLTRANSFERASE 1 controls the level of cholesterol in plants. - Plant Cell 12: 853-870, 2000.

Fowler, S., Thomashow, M.F.: Arabidopsis transcriptome profiling indicates that multiple regulatory pathways are activated during cold acclimation in addition to the CBF cold response pathway. - Plant Cell 14: 1675-1690, 2002.

Haubrich, B.A., Collins, E.K., Howard, A.L., Wang, Q., Snell, W.J., Miller, M.B., Thomas, C.D., Pleasant, S.K., Nes, W.D.: Characterization, mutagenesis and mechanistic analysis of an ancient algal sterol C24-methyltransferase: Implications for understanding sterol evolution in the green lineage. Phytochemistry 113: 64-72, 2015.

Higo, K., Ugawa, Y., Iwamoto, M., Korenaga, T.: Plant cisacting regulatory DNA elements (PLACE) database. - Nucl. Acids Res. 27: 297-300, 1999.

Huang, X.Q., Brûlé-Babel, A. Development of genome-specific primers for homoeologous genes in allopolyploid species: the waxy and starch synthase II genes in allohexaploid wheat (Triticum aestivum L.) as examples. - BMC Res. Notes 3: 140, 2010.

Kagan, R.M., Clarke, S.: Widespread occurrence of three sequence motifs in diverse $\mathrm{S}$-adenosylmethionine-dependent methyltransferases suggests a common structure for these enzymes. - Arch. Biochem. Biophys. 310: 417-427, 1994.

Lescot, M., Déhais, P., Moreau, Y., De Moor, B., Rouzé, P., Rombauts, S.: PlantCARE: a database of plant cis-acting regulatory elements and a portal to tools for in silico analysis of promoter sequences. - Nucl. Acids Res. 30: 325-327, 2002.

Li, C.R., Zhou, Z., Lin, R.X., Zhu, D., Sun, Y.N., Tian, L.L., Li, L., Gao, Y., Wang, S.Q.: $\beta$-Sitosterol decreases irradiationinduced thymocyte early damage by regulation of the intracellular redox balance and maintenance of mitochondrial membrane stability. - J. Cell Biochem. 102: 748-758, 2007.

Ma, J., Stiller, J., Berkman, P.J., Wei, Y., Rogers, J., Feuillet, C., Dolezel, J., Mayer, K.F., Eversole, K., Zheng, Y.L., Liu, C.: Sequence-based analysis of translocations and inversions in bread wheat (Triticum aestivum L.). - PLoS ONE 8: e79329, 
2013.

Marcussen, T., Sandve, S.R., Heier, L., Spannagl, M., Pfeifer, M., Jakobsen, K.S., Wulff, B.B., Steuernagel, B., Mayer, K.F., Olsen, O.A.: Ancient hybridizations among the ancestral genomes of bread wheat. - Science 345: 1250092 , 2014.

Men, S., Boutté, Y., Ikeda, Y., Li, X., Palme, K., Stierhof, Y.D., Hartmann, M.A., Moritz, T., Grebe, M.: Sterol-dependent endocytosis mediates post-cytokinetic acquisition of PIN2 auxin efflux carrier polarity. - Nat. cell. Biol. 10: 237-244, 2008.

Morikawa, T., Mizutani, M., Aoki, N., Watanabe, B., Saga, H., Saito, S., Oikawa, A., Suzuki, H., Sakurai, N., Shibata, D., Wadano, A., Sakata, K., Ohta, D.: Cytochrome $\mathrm{P}_{450}$ CYP710A encodes the sterol C-22 desaturase in Arabidopsis and tomato. - Plant Cell 18: 1008-1022, 2006.

Nakamoto, M., Schmit, A.C., Heintz, D., Schaller, H., Ohta, D.: Diversification of sterol methyltransferase enzymes in plants and a role for $\beta$-sitosterol in oriented cell plate formation and polarized growth. - Plant J. 84: 860-874, 2015.

Neelakandan, A.K., Song, Z., Wang, J., Richards, M.H., Wu, X., Valliyodan, B., Nguyen, H.T., Nes, W.D.: Cloning, functional expression and phylogenetic analysis of plant sterol 24C-methyltransferases involved in sitosterol biosynthesis. - Phytochemistry 70: 1982-1998, 2009.

Neelakandan, A.K., Nguyen, T.M., Kumar, R., Tran, L.S., Guttikonda, S.K., Quach, T.N., Aldrich, D.L., Nes, W.D., Nguyen, H.T.: Molecular characterization and functional analysis of Glycine max sterol methyl transferase 2 genes involved in plant membrane sterol biosynthesis. - Plant mol. Biol. 74: 503-518, 2010.

Nes, W.D. Sterol methyltransferase: enzymology and inhibition. - Biochim. biophys. Acta 1529: 63-88, 2000.

Nes, W.D., Jayasimha, P., Zhou, W., Kanagasabai, R., Jin, C., Jaradat, T.T., Shaw, R.W., Bujnicki, J.M.: Sterol methyltransferase: functional analysis of highly conserved residues by site-directed mutagenesis. - Biochemistry 43: 569-576, 2004.

Nes, W.D., Marshall, J.A., Jia, Z., Jaradat, T.T., Song, Z., Jayasimha, P.: Active site mapping and substrate channeling in the sterol methyltransferase pathway. - J. biol. Chem. 277: 42549-42556, 2002.

Ohta, D., Mizutani, M.: Sterol C22-desaturase and its biological roles. - In: Bach, T.J., Rohme,r M. (ed.): Isoprenoid Synthesis in Plants and Microorganisms: New Concepts and Experimental Approaches. Pp. 381-391. Springer, New York 2013.

Paniagua-Pérez, R., Madrigal-Bujaidar, E., Reyes-Cadena, S., Alvarez-González, I., Sánchez-Chapul, L., Pérez-Gallaga, J., Hernández, N., Flores-Mondragón, G., Velasco, O.: Cell protection induced by betasitosterol: inhibition of genotoxic damage, stimulation of lymphocyte production, and determination of its antioxidant capacity. - Arch. Toxicol. 82: 615-622, 2008.

Paolacci, A.R., Tanzarella, O.A., Porceddu, E., Ciaffi, M.: Identification and validation of reference genes for quantitative RT-PCR normalization in wheat. - BMC mol. Biol. 10:11, 2009.

Papadakis, E., Nicklin, S., Baker, A., White, S.: Promoters and control elements: designing expression cassettes for gene therapy. - Curr. Gene Therapy 4: 89-113, 2004.

Pfaffl, M.W.: A new mathematical model for relative quantification in real-time RT-PCR. - Nucl. Acids Res. 29:
2002-2007, 2001.

Posé, D., Castanedo, I., Borsani, O., Nieto, B., Rosado, A., Taconnat, L., Ferrer, A., Dolan, L., Valpuesta, V., Botella, M.A.: Identification of the Arabidopsis $d r y 2 /$ sqe 1-5 mutant reveals a central role for sterols in drought tolerance and regulation of reactive oxygen species. - Plant J. 59: 63-76, 2009.

Roche, Y., Gerbeau-Pissot, P., Buhot, B., Thomas, D., Bonneau, L., Gresti, J., Mongrand, S., Perrier-Cornet, J.M., SimonPlas, F.: Depletion of phytosterols from the plant plasma membrane provides evidence for disruption of lipid rafts. FASEB J. 22: 3980-3991, 2008.

Ruelland, E., Vaultier, M.N., Zachowski, A., Hurry, V., Kader, J.C., Delseny, M.: Cold signalling and cold acclimation in plants. - Adv. Bot. Res. 49: 35-150, 2009.

Schaeffer, A., Bronner, R., Benveniste, P., Schaller, H.: The ratio of campesterol to sitosterol that modulates growth in Arabidopsis is controlled by STEROL METHYL TRANSFERASE 2 :1. - Plant J. 25: 1-12, 2001.

Schaller, H., Bouvier-Navé, P., Benveniste, P.: Overexpression of an Arabidopsis cDNA encoding a sterol-C24(1)methyltransferase in tobacco modifies the ratio of 24-methyl cholesterol to sitosterol and is associated with growth reduction. - Plant Physiol. 118: 461-469, 1998.

Souter, M.A., Pullen, M.L., Topping, J.F., Zhang, X., Lindsey, $\mathrm{K}$.: Rescue of defective auxin-mediated gene expression and root meristem function by inhibition of ethylene signalling in sterol biosynthesis mutants of Arabidopsis. - Planta 219: 773783, 2004.

Subramaniam, K., Liu, B., Ye, Z., Abbo, S., Ueng, P.P.: Isolation of a gene coding for a putative sterol C-24 methyltransferase in wheat. - Wheat Information Service 89: 17-22, 1999.

Sulkarnayeva, A.G., Valitova, J.N., Mukhitova, F.K., Minibayeva, F.V.: Stress-induced changes in membrane sterols in wheat roots. - Dokl. Biochem. Biophys. 455: 53-55, 2014.

Tang, J., Ohyama, K., Kawaura, K., Hashinokuchi, H., Kamiya, Y., Suzuki, M., Muranaka, T., Ogihara, Y.: A new insight into application for barley chromosome addition lines of common wheat: achievement of stigmasterol accumulation. Plant Physiol. 157: 1555-1567, 2011.

Uemura, M., Steponkus, P.L.: A contrast of the plasma membrane lipid composition of oat and rye leaves in relation to freezing tolerance. - Plant Physiol. 104: 479-496, 1994.

Valitova, J.N., Minibayeva, F.V., Kotlova, E.R., Novikov, A.V., Shavarda, A.L., Murtazina, L.I., Ryzhkina, I.S.: Effects of sterol-binding agent nystatin on wheat roots: the changes in membrane permeability, sterols and glycoceramides. Phytochemistry 72: 1751-1759, 2011.

Valitova, J., Sulkarnayeva, A., Kotlova, E., Ponomareva, A., Mukhitova, F.K., Murtazina, L., Ryzhkina, I., Beckett, R., Minibayeva, F.: Sterol binding by methyl- $\beta$-cyclodextrin and nystatin - comparative analysis of biochemical and physiological consequences for plants. - FEBS J. 281: 20512060, 2014.

Vivancos, M., Moreno, J.J.: Beta-sitosterol modulates antioxidant enzyme response in RAW 264.7 macrophages. Free Radicals Biol. Med. 39: 91-97, 2005.

Wang, H., Nagegowda, D.A., Rawat, R., Bouvier-Navé, P., Guo, D., Bach, T.J., Chye, M.L.: Overexpression of Brassica juncea wild-type and mutant HMG-CoA synthase 1 in Arabidopsis up-regulates genes in sterol biosynthesis and enhances sterol production and stress tolerance. - Plant 
Biotechnol. J. 158: 1789-1802, 2012.

Wendel, J.F.: Genome evolution in polyploids. - Plant mol. Biol. 42: 225-249, 2000.

Willemsen, V., Friml, J., Grebe, M., Van den Toorn, A., Palme, K., Scheres, B.: Cell polarity and PIN protein positioning in Arabidopsis require STEROL METHYLTRANSFERASE1 function. - Plant Cell 15: 612-625, 2003.

Zhang, X., Teixeira da Silva, J.A., Niu, M., Li, M., He, C., Zhao, J., Ma, G.: Physiological and transcriptomic analyses reveal a response mechanism to cold stress in Santalum album L. leaves. - Sci. Rep. 7:42165, 2017. 